\title{
Combined Post-Traumatic Total Aniridia and Glaucoma Management
}

\author{
Eleni Christodoulou ${ }^{a} \quad$ Georgios Batsos $^{b} \quad$ Efstratios Parikakis $^{b}$ \\ Vlasios Papadopoulos ${ }^{a}$ Dimitrios Karagiannis ${ }^{b}$ Panagiotis Petros Koumoutsos ${ }^{b}$ \\ Alexandros Tsilis ${ }^{a}$ Spyridon Drazinos ${ }^{a}$ \\ aOphthalmology Department, Corfu General Hospital, Corfu, Greece; b2nd Ophthalmology \\ Department, Ophthalmiatreio Eye Hospital of Athens, Athens, Greece
}

\author{
Keywords \\ Ocular trauma $\cdot$ Aniridia $\cdot$ Glaucoma $\cdot$ Vitrectomy
}

\begin{abstract}
We report a novel approach for managing traumatic total aniridia associated with secondary glaucoma. A 65-year-old male with symptoms of blurred vision, glare, and photophobia was managed in a district general hospital for post-traumatic aniridia associated with secondary glaucoma affecting the right eye. The patient previously suffered blunt ocular injury associated with rhegmatogenous retinal detachment, which was treated with pars plana vitrectomy and silicone oil (SO) tamponade and subsequent removal of SO (ROSO). Combined trabeculectomy and implantation of a foldable prosthetic iris (Reper NN Ltd) successfully addressed the dual pathology of aniridia and secondary glaucoma. In summary, the utilization of the trabeculectomy fistula can serve as the implant insertion site, which minimizes the requirement for additional incisions.
\end{abstract}

\section{Introduction}

Blunt ocular trauma can result in severe complications such as rhaegmatogenous retinal detachment (RRD), iris defects, and glaucoma [1,2]. Besides any cosmetic considerations, iris defects can cause various symptoms such as glare, photophobia, loss of contrast sensitivity, or decreased visual acuity $[3,4]$. Some of these symptoms may be addressed with the aid of coloured contact lenses, corneal tattooing, or prosthetic iris devices $[2,4]$. Secondary glaucoma can develop due to the trauma, but there has been a series of case reports regarding silicone oil (SO) induced glaucoma or post iris implantation glaucoma $[5,6]$. What follows are the 
Christodoulou et al.: Combined Post-Traumatic Total Aniridia and Glaucoma Management

salient points of the management of post-traumatic total aniridia associated with secondary glaucoma in a pseudophakic eye.

\section{Case Presentation}

A 65-year-old male with symptoms of blurred vision, glare, and photophobia was referred to a district general hospital for the management of post-traumatic aniridia associated with glaucoma affecting his right eye. The patient suffered a closed globe injury associated with right RRD. Six months before the trauma, the patient underwent uneventful bilateral phacoemulsification. The primary management of the RRD took place in a tertiary centre and included pars plana vitrectomy and silicone oil (SO) tamponade. Following the initial procedure, the best-corrected visual acuity was $6 / 240$ in his right eye and $6 / 6$ in his left. The intraocular pressure (IOP) was 30 and $15 \mathrm{~mm} \mathrm{Hg}$ in his right and left eye, respectively, without ocular hypotensive agents. Slit-lamp examination showed corneal haze and total iris absence associated with partial ( $<2$ clock $\mathrm{h}$ ) loss of zonules. The intraocular lens (IOL) was inside the capsular bag, centred, and not tilted. There was evidence of keratic precipitates and the accumulation of SO droplets (fish eggs) in the anterior chamber. Fundal examination revealed a flat retina with lattice degeneration. There were no signs of proliferative vitreoretinopathy. The cup to disk (C/D) ratio was 0.8 and 0.3 in his right and left eye, respectively. Glaucoma medication (dorzolamide-timolol combination), combined with dexamethasone drops, was commenced. Two weeks later, the patient underwent 360 prophylactic laser retinopexy. Three weeks following the prophylactic laser, the corneal haze improved significantly, and the patient underwent removal of SO (ROSO). Figure 1a shows the beginning of the operation.

During the 2 months following ROSO, IOP was $18 \mathrm{~mm} \mathrm{Hg}$ with (dorzolamide-timolol combination). Unfortunately, the IOP gradually increased to $30 \mathrm{~mm} \mathrm{Hg}$ and became refractive to antiglaucoma medications. The patient was complaining of increasing glare and photophobia.

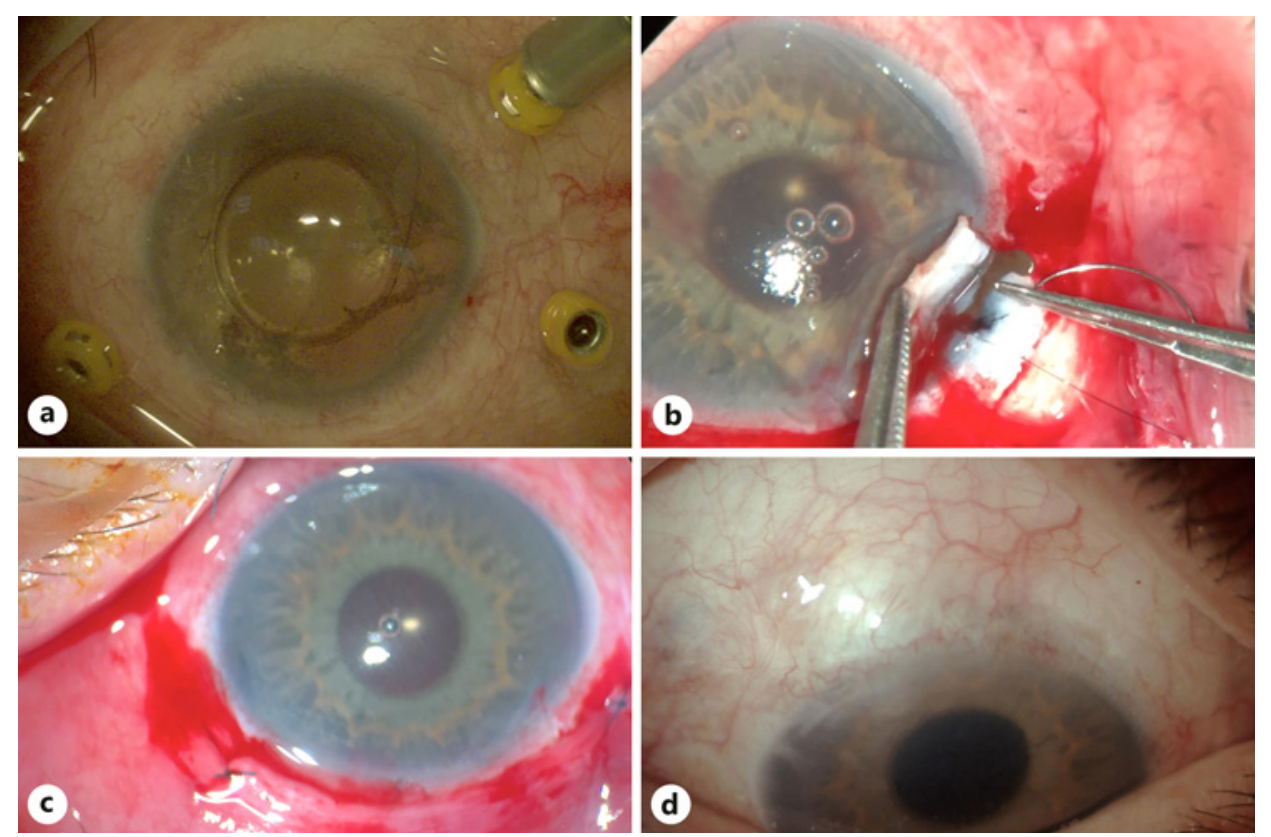

Fig. 1. Stages from 2 operations and final result. Beginning of SO extraction (a), implantation of artificial iris ( 1 of the 3 haptics is externalized) (b), end of the operation (c), final result after 3 months (artificial iris in proper position and a functional filtering bleb) (d). SO, silicone oil. 
Following a comprehensive risk assessment and informed patient's consent, there was a joint agreement to proceed to combined prosthetic iris implantation (Reper NN Ltd) with glaucoma filtration surgery. Coloured photos of the fellow eye were taken and matched through the sample book of Reper NN Ltd. The photos were also sent to the manufacturer for cross check.

The operation began with a limbus-based conjunctival flap preparation, followed by a scleral rectangular flap creation. The next step included the creation of scleral tunnel (fistula) and the sclerostomy (with a punch). The artificial iris was folded and inserted through the tunnel (fistula) (shown in Fig. 1b). Following insertion, the implant was sutured to the sclera, in an adjacent to the flap position, with 10.0 nylon suture. Prior to the implant insertion, the suture was passed through one of the 3 haptic holes. Finally, the scleral flap was sutured. The operation was completed with the conjunctival closure. The end of the operation is shown in Figure 1c.

Three months after the procedure, the patient's IOP was $15 \mathrm{~mm} \mathrm{Hg}$ without medication and the best-corrected visual acuity was $6 / 60$. The cornea was clear. There were no further post-operative complications. The patient denied any glare or photophobia. Most importantly, he was pleased with the cosmetic outcome (shown in Fig. 1d).

Cases of post-traumatic total aniridia with simultaneous IOL and capsular bag preservation have been reported before [7-10]. An existing corneal incision (post-cataract surgery) can serve as a decompression valve reducing the risk of globe rupture [10]. Yet, the risk of complications such as RRD remains, prompting physicians for meticulous examination.

Our patient was concerned about the functional and cosmetic issues caused by the total aniridia. The patient's expectations included a comprehensive and long-lasting solution. Current anterior chamber devices for subtotal or total iris defects include the iris-lens diaphragm; the tension ring-based prosthetic iris and the customizable artificial iris prosthesis [4]. In this case, the IOL was central in situ, and the capsular bag was intact, and only a partial zonular loss was present. Therefore, the implantation of a foldable artificial iris, which requires a small incision and offers a customised colour design, was considered by the surgical team as the most feasible surgical approach.

In this case, the Reper NN Ltd (model C) artificial iris was chosen. This model is a foldable acrylic artificial implant available in 3 different models and 300 different colour designs. The option of tailor-made customised iris pattern constitutes a significant advantage of the implant. The model C has 3 haptics for better centration and stabilization.

This patient had advanced glaucomatous disc cupping with C/D 0.8, in this right eye. After the ROSO, antiglaucoma medical treatment did not lower the IOP; therefore, a filtration surgery was required. This patient had already been operated 3 times (phacoemulsification, pars plana vitrectomy, and ROSO) in the same eye, and so the idea of a combined approach was more desirable. The main advantage of this approach is that the trabeculectomy fistula can conveniently serve as the iris implant incision, avoiding additional corneal wounds. A similar technique has been described for implanting an IOL during combined phacoemulsification and trabeculectomy [11]. To our knowledge, the combined technique of trabeculectomy and prosthetic iris implantation has not been previously reported.

\section{Conclusion}

This case report makes 2 main contributions in the field of ophthalmic surgery. First, it blurs the borders between anterior segment reconstruction and penetrating glaucoma surgery. Second, this report provides the salient points of a novel surgical approach. Foldable artificial iris can provide good functional and cosmetic results.

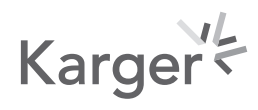


Christodoulou et al.: Combined Post-Traumatic Total Aniridia and Glaucoma Management

\section{Statement of Ethics}

The patient has given written informed consent for the publishing of medical record information and accompanying images. The assessment of the case was in accordance with the Declaration of Helsinki.

\section{Conflict of Interest Statement}

The authors have no conflicts of interest to declare.

\section{Funding Sources}

The authors received no financial support for the research, authorship, or publication of this article.

\section{Author Contributions}

Eleni Christodoulou and Georgios Batsos: acquisition and analysis of data for the work. Drafting the work. Efstratios Parikakis, Vlasios Papadopoulos, and Dimitrios Karagiannis: interpretation of data for the work. Revision of work. Panagiotis Petros Koumoutsos and Alexandros Tsilis: interpretation of data for the work. Drafting the work. Spyridon Drazinos: conception, design, and acquisition of the work. Revision of work. All authors: final approval of the version to be published. Agreement to be accountable for all aspects of the work in ensuring that questions related to the accuracy or integrity of any part of the work are appropriately investigated and resolved.

\section{References}

1 Viestenz A, Küchle M. [Blunt ocular trauma. Part I: blunt anterior segment trauma]. Ophthalmologe. 2004 Dec; 101(12):1239-8; quiz 57-8.

2 Mikhail M, Koushan K, Sharda RK, Isaza G, Mann KD. Traumatic aniridia in a pseudophakic patient 6 years following surgery. Clin Ophthalmol. 2012;6:237-41.

3 Beekhuis WH, Drost BH, van der Velden/Samderubun EM. A new treatment for photophobia in posttraumatic aniridia: a case report. Cornea. 1998 May;17(3):338-41.

4 Srinivasan S, Ting DS, Snyder ME, Prasad S, Koch HR. Prosthetic iris devices. Can J Ophthalmol. 2014 Feb;49(1): 6-17.

5 Ichhpujani P, Jindal A, Jay Katz L. Silicone oil induced glaucoma: a review. Graefes Arch Clin Exp Ophthalmol. 2009 Dec;247(12):1585-93.

6 Mayer CS, Laubichler AE, Khoramnia R, Tandogan T, Prahs P, Zapp D, et al. Challenges and complication management in novel artificial Iris implantation. J Ophthalmol. 2018;2018:3262068.

7 Ball J, Caesar R, Choudhuri D. Mystery of the vanishing iris. J Cataract Refract Surg. 2002 Jan;28(1):180-1.

8 Muzaffar W, O’Duffy D. Traumatic aniridia in a pseudophakic eye. J Cataract Refract Surg. 2006 Feb;32(2): 361-2.

9 Kim KH, Kim WS. Aniridia after blunt trauma and presumed wound dehiscence in a pseudophakic eye. Arq Bras Oftalmol. 2016 Feb; 79(1):44-5.

10 Sophocleous S. Doctor, where is my iris? BMJ Case Rep. 2016 May 52016;2016.

11 Dada T, Muralidhar R, Sethi HS. Insertion of a foldable hydrophobic IOL through the trabeculectomy fistula in cases with microincision cataract surgery combined with trabeculectomy. BMC Ophthalmol. 2006 Mar 19;6:14.

\section{Karger'₹}

